Supplementary Material

\title{
An Expedient Enantioselective Route to Diaminotetralins: Application in the Preparation of Analgesic Compounds
}

\author{
Mark Lautens*, Keith Fagnou and Valentin Zunic \\ Davenport Research Laboratories, University of Toronto, Department of Chemistry \\ 80 St. George St., Toronto, Ontario, Canada M5H 3H6 \\ mlautens@chem.utoronto.ca
}

The following general experimental details apply to all following reactions.

All flasks were flame-dried under a stream of nitrogen or argon and cooled before use. Solvents and solutions were transferred with syringes and cannulae using standard inert atmosphere techniques.

${ }^{1} \mathrm{H}$ NMR spectra were recorded at $300 \mathrm{MHz}$ using a Varian Gemini NMR spectrometer or at $400 \mathrm{MHz}$ using a Varian XL400 spectrometer with $\mathrm{CDCl}_{3}$ as reference standard $(\delta 7.24$ $\mathrm{ppm}$ ) or some other suitable solvent. Spectral features are tabulated in the following order: chemical shift $(\delta, \mathrm{ppm})$; number of protons; multiplicity (s-singlet, d-doublet, $\mathrm{t}$ triplet, q-quartet, m-complex multiplet, br-broad); coupling constants $(J, \mathrm{~Hz}) .{ }^{13} \mathrm{C}$ NMR spectra were recorded at $400 \mathrm{MHz}$ with $\mathrm{CDCl}_{3}$ as reference standard $(\delta=77.0 \mathrm{ppm})$ or some other suitable solvent.

IR spectra were obtained using a Nicolet DX FT-JR spectrometer as a $\mathrm{KBr}$ pellet or neat film between $\mathrm{KBr}$ plates. High resolution mass spectra were obtained from a VG 70-250S (double focusing) mass spectrometer at $70 \mathrm{eV}$. Combustion analyses were submitted to Canadian Microanalytical Service Ltd., BC. Optical rotations were measured on a PerkinElmer Model 243 Polarimeter using the sodium D line with spectro-grade $\mathrm{CHCl}_{3}$ in a 1 $\mathrm{dm}$ cell. Melting points were taken on a Fisher-Johns melting point apparatus and are uncorrected.

HPLC analysis was performed on a Waters 600E with Chiralcel OD or OJ columns. Analytical TLC was performed using EM Separations precoated silica gel $0.2 \mathrm{~mm}$ layer UV 254 fluorescent sheets. Column chromatography was performed as "Flash Chromatography" as reported by Still ${ }^{1}$ using (200-400 mesh) Merck grade silica gel.

Diethyl ether, THF, benzene and toluene were distilled from sodium benzophenone ketyl immediately prior to use. $\mathrm{CH}_{2} \mathrm{Cl}_{2}$ was distilled from calcium hydride. DME was distilled from sodium benzophenone ketyl and stored. DMF was dried and stored over activated molecular sieves. The (R, S)-PPF-P $\mathrm{Pu}_{2}$ ligand was donated by Solvias. $\left(\alpha \mathrm{S}, \alpha^{1} \mathrm{~S}\right)-2,2^{1}-$ $\operatorname{Bis}\left(\alpha-N, N\right.$-dimethylaminoethyl)-(R,R)-1, $1^{1}$-bis-(diphenylphosphino)-ferrocene $\quad\left(\mathrm{C}_{2}\right.$ -

'Still, W.C.; Kahn, M.; Mitra, A. J. Org. Chem. 1989, 54, 5667. 
Ferriphos) was prepared according to literature procedure ${ }^{2}$ starting from (R)-2-MethylCBS-oxazaborolidine. All other reagents were obtained from Aldrich and used as received unless otherwise stated.

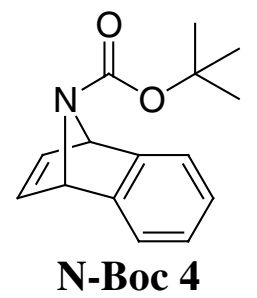

1,4-Dihydro-1,4-epiazano-naphthalene-9-carboxylic acid tert-butyl ester (N-Boc 4): To $N$-Boc pyrrole $(16.7 \mathrm{~g}, 100 \mathrm{mmol})$ in DME $(40 \mathrm{~mL})$ at $50^{\circ} \mathrm{C}$ in a flame dried 3-neck flask fitted with a reflux condenser and 2 addition funnels was simultaneously added a solution of anthranilic acid $(13.8 \mathrm{~g}, 100 \mathrm{mmol})$ in DME $(\sim 100 \mathrm{~mL})$ and a separate solution of isoamylnitrite $(16.8 \mathrm{~mL}, 125 \mathrm{mmol})$ in DME $(50 \mathrm{~mL})$. The addition took about 45 minutes. The reaction was allowed to stir at $50^{\circ} \mathrm{C}$ for $30 \mathrm{~mm}$ until no further gas was evolved. The crude red solution was reduced in volume by rotovap distillation to approximately half the original volume. The reaction mixture was then partitioned between $\mathrm{Et}_{2} \mathrm{O}$ and saturated $\mathrm{K}_{2} \mathrm{CO}_{3}$ and the aqueous layer was extracted 3 times with $\mathrm{Et}_{2} \mathrm{O}$. The combined organic layers were washed with brine, dried over $\mathrm{MgSO}_{4}$ and concentrated. The resulting dark brown oil was further evaporated under reduce pressure to remove the iosamyalcohol byproduct. This crude mixture can be purified by repeated recrystalization from hexanes or the process can be sped up by performing column chromatography with a short plug of silica gel to remove most of the colored byproducts. Recrystalization gives N-Boc 4 in $63 \%$ yield as an off white solid. Spectral data is identical to that reported in the literature. ${ }^{3}$

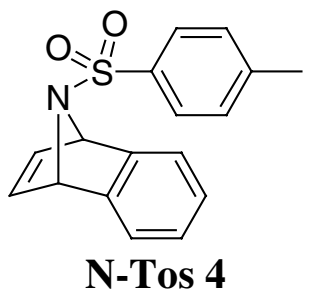

9-(Toluene-4-sulfonyl)-1,4-dihydro-1,4-epiazano-naphthalene (N-Tos 4): N-Boc 4 (6

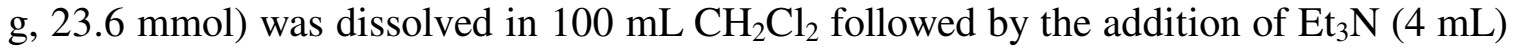
and heating to reflux. TMSI $(3.7 \mathrm{~mL}, 25.9 \mathrm{mmol})$ was added dropwise over 10 minutes and the reaction was heated at reflux for an additional 15 minutes after which time TLC analysis showed complete consumption of $\mathbf{N}$-Boc 4 . The reaction mixture was cooled to $0^{\circ} \mathrm{C}$ then $\mathrm{MeOH}(1 \mathrm{~mL}, 30 \mathrm{mmol})$ was added dropwise. After 10 minutes at $0^{\circ} \mathrm{C}$, Tosyl chloride $(4.56 \mathrm{~g}, 24 \mathrm{mmol})$ was added and the reaction was allowed to warm to room temperature. The reaction was stirred at room temperature for an additional hour. Water $(100 \mathrm{~mL})$ and $\mathrm{CH}_{2} \mathrm{Cl}_{2}$ was added until all precipitates dissolved. The organic and

\footnotetext{
${ }^{2}$ Schwink, L.; Knochel, P. Chem. Eur. J. 1998, 4, No.5, 950.

${ }^{3}$ Carpino, L.A.; Barr, D.E. J. Org. Chem. 1966, 31, 764.
} 
aqueous phases were separated and the aqueous phase was extracted twice with $\mathrm{CH}_{2} \mathrm{Cl}_{2}$. The organic fractions were combined, dried over $\mathrm{MgSO}_{4}$ and concentrated. Recrystalization from ethylacetate gave $\mathbf{N}$-Tos $\mathbf{4}$ as a crystalline solid. Spectral data was identical to that reported in the literature. ${ }^{4}$

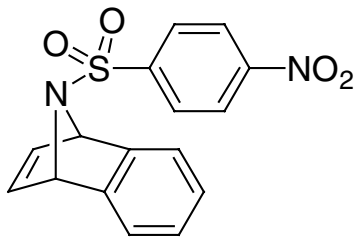

N-Nos 4

9-(4-Nitro-benzenesulfonyl)-1,4-dihydro-1,4-epiazano-naphthalene (N-Nos 4): $\boldsymbol{N}$-Boc $4(6 \mathrm{~g}, 23.6 \mathrm{mmol})$ was dissolved in $100 \mathrm{~mL} \mathrm{CH}_{2} \mathrm{Cl}_{2}$ followed by the addition of $\mathrm{Et}_{3} \mathrm{~N}$ (4 $\mathrm{mL})$ and heating to reflux. TMSI $(3.7 \mathrm{~mL}, 25.9 \mathrm{mmol})$ was added dropwise over 10 minutes and the reaction was heated at reflux for an additional 15 minutes after which time TLC analysis showed complete consumption of N-Boc 4. The reaction mixture was cooled to $0^{\circ} \mathrm{C}$ then $\mathrm{MeOH}(1 \mathrm{~mL}, 30 \mathrm{mmol})$ was added dropwise. After 10 minutes at $0^{\circ} \mathrm{C}$, Nosyl chloride $(5.5 \mathrm{~g}, 24 \mathrm{mmol})$ was added and the reaction was allowed to warm to room temperature. The formation of a white precipitate was observed at this point. The reaction was stirred at room temperature for an additional hour. Water $(100 \mathrm{~mL})$ and $\mathrm{CH}_{2} \mathrm{Cl}_{2}$ was added until all precipitates dissolved. The organic and aqueous phases were separated and the aqueous phase was extracted twice with $\mathrm{CH}_{2} \mathrm{Cl}_{2}$. The organic fractions were combined, dried over $\mathrm{MgSO}_{4}$ and concentrated. Trituration in $\mathrm{Et}_{2} \mathrm{O}$ gave $7.13 \mathrm{~g}$ of a white crystalline solid N-Nos 4 (92\% yield). $\quad R_{f}=0.45$ on silica gel (40\% ethyl acetate:hexanes); mp $205-210^{\circ} \mathrm{C}$ (decomp) $\left(\mathrm{Et}_{2} \mathrm{O}\right)$; IR $\left(\mathrm{KBr}, \mathrm{cm}^{-1}\right){ }^{1} \mathrm{H}$ NMR $(400 \mathrm{MHz}$, $d_{6}$-DMSO) $\delta 8.15(2 \mathrm{H}, \mathrm{d}, J=7.9 \mathrm{~Hz}), 7.76(2 \mathrm{H}, \mathrm{d}, J=7.9 \mathrm{~Hz}), 7.12-7.05(2 \mathrm{H}, \mathrm{m}), 6.90$ $(2 \mathrm{H}, \mathrm{s}), 6.73-6.66(2 \mathrm{H}, \mathrm{m}), 5.64(2 \mathrm{H}, \mathrm{s}) ;{ }^{13} \mathrm{C} \mathrm{NMR}\left(400 \mathrm{MHz}, d_{6}\right.$-DMSO) $\delta 149.6,147.1$, 143.2, 142.9, 129.9, 124.9, 124.1, 121.7, 67.5. Anal. Calcd for $\mathrm{C}_{16} \mathrm{H}_{12} \mathrm{~N}_{2} \mathrm{O}_{4} \mathrm{~S}$ : C, 58.53; H, 3.68; N, 8.53. Found: C, 58.55; H, 7.10; N, 5.67.

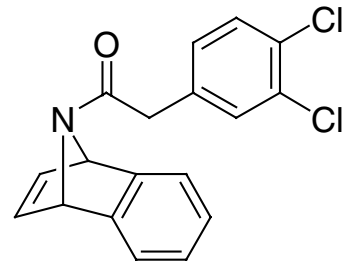

2-(3,4-Dichloro-phenyl)-1-(1,4 dihydro-1,4-epizano-naphthalen-9yl)-ethanone : $\mathbf{N}$ -

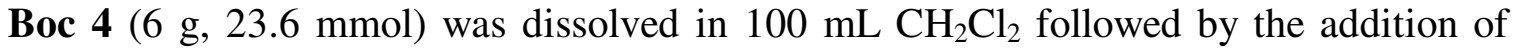
$\mathrm{Et}_{3} \mathrm{~N}(4 \mathrm{~mL})$ and heating to reflux. TMSI $(3.7 \mathrm{~mL}, 25.9 \mathrm{mmol})$ was added dropwise over 10 minutes and the reaction was heated at reflux for an additional 15 minutes after which time TLC analysis showed complete consumption of $\mathbf{N}$-Boc 4. The reaction mixture was cooled to $0^{\circ} \mathrm{C}$ then $\mathrm{MeOH}(1 \mathrm{~mL}, 30 \mathrm{mmol})$ was added dropwise. After 10 minutes at $0^{\circ} \mathrm{C}, 3,4$-Diclorophenyl acetyl chloride $(5.3 \mathrm{~g}, 24 \mathrm{mmol})$ was added and the reaction was

\footnotetext{
${ }^{4}$ Kaupp, G. Chem. Ber. 1970, 2288.
} 
allowed to warm to room temperature. The reaction was stirred at room temperature for an additional hour. Water $(100 \mathrm{~mL})$ and $\mathrm{CH}_{2} \mathrm{Cl}_{2}$ was added until all precipitates dissolved. The organic and aqueous phases were separated and the aqueous phase was extracted twice with $\mathrm{CH}_{2} \mathrm{Cl}_{2}$. The organic fractions were combined, dried over $\mathrm{MgSO}_{4}$ and concentrated. Trituration in $\mathrm{Et}_{2} \mathrm{O}$ gave $7.13 \mathrm{~g}$ of a white crystalline solid $(92 \%$ yield). $\quad \mathrm{R}_{\mathrm{f}}=0.45$ on silica gel (20\% ethyl acetate:hexanes); $\mathrm{mp} 124-125^{\circ} \mathrm{C}\left(\mathrm{Et}_{2} \mathrm{O}\right) ; \mathrm{IR}$ $\left(\mathrm{KBr}, \mathrm{cm}^{-1}\right)$ 3691(w), 3050(s), 2685(w), 2528(w), 2305(m), 2169(w), 2119(w), 1996(w), 1794(m), 1648(s), 1560(w), 1421(s), 1262(s), 1133(w), 1034(m), 906(s), 730(s); ${ }^{1} \mathrm{H}$ NMR $\left(400 \mathrm{MHz}, \mathrm{CDCl}_{3}\right) \delta$ 7.30-7.22(3H, m), 7.12-7.02(2H, m), 6.97-6.88(4H, m), 5.89 $(1 \mathrm{H}, \mathrm{s}), 5.52(1 \mathrm{H}, \mathrm{s}), 3.55-3.47(2 \mathrm{H}, \mathrm{m}) ;{ }^{13} \mathrm{C} \mathrm{NMR}\left(400 \mathrm{MHz}, \mathrm{CDCl}_{3}\right) \delta 165.25$, $147.98,147.66,144.19,142.20,134.24,132.51,131.12,130.45,128.61,125.47,125.20$, 121.58, 120.34, 65.90, 63.65, 39.99. HRMS calcd for $\mathrm{C}_{18} \mathrm{H}_{13} \mathrm{NOCl}_{2}\left(\mathrm{M}^{+}\right): 329.0374$. Found: 329.0367.

General Procedure for the ARO Reactions with Amine Nucleophiles: To a flame dried round bottom flask were added N-Boc $4(100 \mathrm{mg}, 0.41 \mathrm{mmol}),[\mathrm{Rh}(\mathrm{COD}) \mathrm{Cl}]_{2}$ (2.5mol\%), $(R, S)$-PPF-P ${ }^{\mathrm{t}} \mathrm{Bu}_{2}$ or $\mathrm{C}_{2}$-Ferriphos $(10 \mathrm{~mol} \%)$ followed by addition of THP and nucleophile (3 equivalents) The mixture was heated to $100^{\circ} \mathrm{C}$ until the reaction was complete as judged by thin layer chromatography. The solvents were removed in vacuo and the crude mixture was purified by flash chromatography.

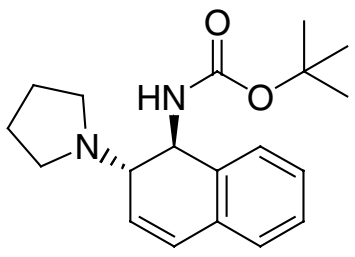

(5b)

(1S, 2S)-(2-Pyrrolidin-1-yl-1,2-dihydro-naphthalen-1-yl)-carbamic acid tert-butyl ester $[(+)-5 b]$.

Following the general procedure, $\mathbf{5 b}$ was obtained as a white solid (99mg, 77\%). The ee was determined to be $86 \%$ using HPLC analysis on a CHIRALCEL AD column, $\lambda=486$ $\mathrm{nm}$. Retention times in $2 \%$ isopropanol in hexanes $(0.5 \mathrm{ml} / \mathrm{min}, 15 \mathrm{C})$ were $21.45 \mathrm{~min}$ (minor) and $23.04 \mathrm{~min}$ (major). $\mathrm{R}_{\mathrm{f}}=0.2$ on silica gel (50\% ethyl acetate:hexanes); $\mathrm{mp} 84$ $87^{\circ} \mathrm{C}\left(\mathrm{Et}_{2} \mathrm{O}\right) ;[\alpha]^{25}{ }_{\mathrm{D}}=+230.5\left(\mathrm{c}=0.98, \mathrm{CCl}_{4}\right) ; \mathrm{IR}\left(\mathrm{KBr}, \mathrm{cm}^{-1}\right) 3450(\mathrm{w}), 2809(\mathrm{~m}), 2360(\mathrm{~s})$, 1716(s), 1653(w), 1557(s), 1251(m), $1169(\mathrm{~m}), 1005(\mathrm{~m}) ;{ }^{1} \mathrm{H}$ NMR $\left(400 \mathrm{MHz}, \mathrm{CDCl}_{3}\right) \delta$ 7.33-7.31(1H, m), 7.24-7.17(2H, m), 7.08-7.06 $(1 \mathrm{H}, \mathrm{m}), 6.60(1 \mathrm{H}, \mathrm{d}, \mathrm{J}=9.6 \mathrm{~Hz}), 5.97(1 \mathrm{H}$, $\mathrm{dd}, \mathrm{J}=9.8 \mathrm{~Hz}, 5.2 \mathrm{~Hz}), 4.93(1 \mathrm{H}, \mathrm{br} \mathrm{s}), 4.76(1 \mathrm{H}, \mathrm{br} \mathrm{d}, \mathrm{J}=6 \mathrm{~Hz}), 3.34(1 \mathrm{H}, \mathrm{t}, \mathrm{J}=4 \mathrm{~Hz}), 2.69-$ $2.59(4 \mathrm{H}, \mathrm{m}), 1.68(4 \mathrm{H}, \mathrm{m}), 1.42(9 \mathrm{H}, \mathrm{s}) ;{ }^{13} \mathrm{C} \mathrm{NMR}\left(400 \mathrm{MHz}, \mathrm{CDCl}_{3}\right) \delta 155.15,134.86$, $132.17,129.18,128.42,128.14,126.78,126.23,61.23,50.12,28.43,23.48$. HRMS calcd for $\mathrm{C}_{19} \mathrm{H}_{26} \mathrm{~N}_{2} \mathrm{O}_{2}\left(\mathrm{M}^{+}\right)$: 314.1994. Found: 314.1991. 


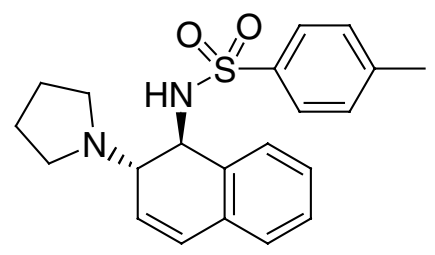

$(+/-) 5 \mathbf{a}$

4-Methyl-N-(2-pyrrolidin-1-yl-1,2-dihydro-naphthalen-1-yl)-benzenesulfonamide [(+/-) 5a]: To round bottomed flask was added N-Tos 4 (60 mg, $0.2 \mathrm{mmol})$, $[\mathrm{Rh}(\mathrm{COD}) \mathrm{Cl}]_{2}(2.5 \mathrm{mg}, 0.005 \mathrm{mmol})$, and DPPF (5.5 mg, $\left.0.01 \mathrm{mmol}\right)$. THF (2 ml) was then added followed by triethylamine hydrochloride $(138 \mathrm{mg}, 1.0 \mathrm{mmol})$ and pyrrolidine $(83 \mu \mathrm{l}, 1.0 \mathrm{mmol})$. The resulting heterogeneous mixture was heated to reflux for 14 hours. Upon completion, the reaction mixture was concentrated and chromatographed (30\% ethyl acetate:hexanes) to give (+/-) 5a (70 $\mathrm{mg}, 96 \%)$ a white solid. The regiochemistry and relative stereochemistry was proven by $\mathrm{X}$-ray diffraction. $\mathrm{R}_{\mathrm{f}}=0.15$ on silica gel (30\% ethyl acetate:hexanes); mp 140-145 ${ }^{\circ}$; IR (KBr, cm $\left.{ }^{-1}\right) 3496$ (br), 3035 (m), 2967 (s), 1454 (m), 1193 (s), 1117 (m), 1048 (s). ${ }^{1} \mathrm{H}$ NMR (400MHz, CDCl $\left.\mathrm{C}_{3}\right) \delta$ $7.74(2 \mathrm{H}, \mathrm{d}, J=8.3 \mathrm{~Hz}), 7.30(2 \mathrm{H}, \mathrm{d}, J=8.2 \mathrm{~Hz}), 7.22-7.17(1 \mathrm{H}, \mathrm{m}), 7.08-7.02(2 \mathrm{H}, \mathrm{m})$, $6.84(1 \mathrm{H}, \mathrm{d}, J=7.5 \mathrm{~Hz}), 6.61(1 \mathrm{H}, \mathrm{d}, J=9.7 \mathrm{~Hz}), 5.93(1 \mathrm{H}, \mathrm{dd}, J=4.9,9.7 \mathrm{~Hz}), 4.70(1 \mathrm{H}, \mathrm{br}$ s), $4.45(1 \mathrm{H}, \mathrm{d}, J=3.7 \mathrm{~Hz}), 3.89(1 \mathrm{H}, \mathrm{dd}, J=4.2,4.2 \mathrm{~Hz}), 2.58-2.49(2 \mathrm{H}, \mathrm{m}), 2.45(3 \mathrm{H}, \mathrm{s})$, 2.36-2.29 (2H, m), 1.63-1.58 (4H, m); $\left.{ }^{13} \mathrm{C} \mathrm{NMR} \mathrm{(400MHz,} \mathrm{CDCl}_{3}\right) \delta 143.46,138.05$, $133.63,132.17,129.72,129.46,128.55,128.27,128.16,127.40,126.91,125.59,61.24$, 53.63, 49.97, 23.46, 21.65. Anal. Calcd for $\mathrm{C}_{21} \mathrm{H}_{24} \mathrm{~N}_{2} \mathrm{O}_{2} \mathrm{~S}: \mathrm{C}, 68.45 ; \mathrm{H}, 6.56 ; \mathrm{N}, 7.60$. Found: C, 68.51; H, 6.62; N, 7.55.

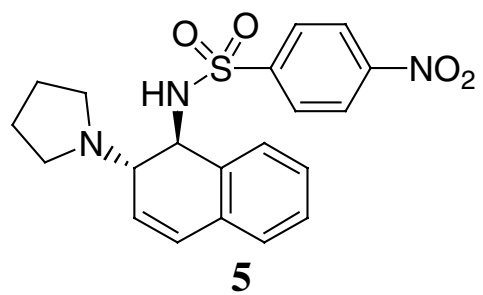

4-Nitro-N-(2-pyrrolidin-1-yl-1,2-dihydro-naphthalen-1-yl)-benzenesulfonamide (5): To round bottomed flask was added $\mathbf{N}-\mathbf{N o s} 4(66 \mathrm{mg}, 0.2 \mathrm{mmol})$, [Rh(COD)Cl $]_{2}(2.5 \mathrm{mg}$, $0.005 \mathrm{mmol})$, and DPPF $(5.5 \mathrm{mg}, 0.01 \mathrm{mmol})$. THF $(2 \mathrm{ml})$ was then added followed by triethylamine hydrochloride $(138 \mathrm{mg}, 1.0 \mathrm{mmol})$ and pyrrolidine $(83 \mu \mathrm{l}, 1.0 \mathrm{mmol})$. The resulting heterogeneous mixture was heated to reflux for 16 hours. Upon completion, the reaction mixture was concentrated and chromatographed (30\% ethyl acetate:hexanes) to give $5(67 \mathrm{mg}, 84 \%)$ a white solid. $\mathrm{R}_{\mathrm{f}}=0.12$ on silica gel (30\% ethyl acetate:hexanes); mp 142-145 C; IR (KBr, cm ${ }^{-1}$ ) 3389 (br), 3026 (m), 2978 (s), 1497 (s), 1343 (s), 1117 (m), 1048 (s). ${ }^{1} \mathrm{H}$ NMR $\left(400 \mathrm{MHz}, \mathrm{CDCl}_{3}\right) \delta 8.30(2 \mathrm{H}, \mathrm{d}, J=8.8 \mathrm{~Hz}), 7.99(2 \mathrm{H}, \mathrm{d}, J=$ $8.8 \mathrm{~Hz}), 7.24-7.18(1 \mathrm{H}, \mathrm{m}), 7.10-7.04(2 \mathrm{H}, \mathrm{m}), 6.95-6.90(1 \mathrm{H}, \mathrm{m}), 6.63(1 \mathrm{H}, \mathrm{d}, J=9.9 \mathrm{~Hz})$, $5.93(1 \mathrm{H}, \mathrm{dd}, J=4.7,9.7 \mathrm{~Hz}), 5.20-4.80(1 \mathrm{H}$, br s $), 4.60(1 \mathrm{H}, \mathrm{d}, J=3.8 \mathrm{~Hz}), 3.40-3.35(1 \mathrm{H}$, $\mathrm{m}), 2.58-2.50(2 \mathrm{H}, \mathrm{m}), 2.43-2.34(2 \mathrm{H}, \mathrm{m}), 1.64-1.57(4 \mathrm{H}, \mathrm{m}) ;{ }^{13} \mathrm{C}$ NMR $(400 \mathrm{MHz}$, $\left.\mathrm{CDCl}_{3}\right) \delta 149.8,147.1,132.8,131.9,129.7,128.8,128.2,128.1,128.0,127.1,125.0$, 124.1, 61.4, 54.4, 50.0, 23.4. Anal. Calcd for $\mathrm{C}_{20} \mathrm{H}_{21} \mathrm{~N}_{3} \mathrm{O}_{4} \mathrm{~S}: \mathrm{C}, 60.13 ; \mathrm{H}, 5.30 ; \mathrm{N}, 10.52$. Found: C, 60.16; H, 5.33; N, 10.50 . 


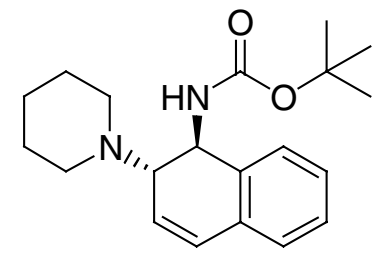

(7)

(1S, 2S)-(2-Piperidin-1-yl-1,2-dihydro-naphthalen-1-yl)-carbamic acid tert-butyl ester (7)

Following the general procedure, 7 was obtained as a white solid (94mg, 70\%). The ee was determined to be $84 \%$ using HPLC analysis on a CHIRALCEL OD column, $\lambda=486$ $\mathrm{nm}$. Retention times in $2 \%$ isopropanol in hexanes were $5.61 \mathrm{~min}$ (minor) and $6.02 \mathrm{~min}$ (major). $\quad \mathrm{R}_{\mathrm{f}}=0.3$ on silica gel (40\% ethyl acetate:hexanes); $\mathrm{mp} 118-122^{\circ} \mathrm{C}\left(\mathrm{Et}_{2} \mathrm{O}\right)$; $[\alpha]^{25}{ }_{\mathrm{D}}=+200.5\left(\mathrm{c}=0.98, \mathrm{CCl}_{4}\right) ; \quad \mathrm{IR}\left(\mathrm{KBr}, \mathrm{cm}^{-1}\right) 3448(\mathrm{~m}), 2978(\mathrm{~m}), 2288(\mathrm{~m}), 1713(\mathrm{~s})$, 1549(s), 1251(s), 1170(m), 1005(m), 760(s); ${ }^{1} \mathrm{H}$ NMR (400MHz, $\left.\mathrm{CDCl}_{3}\right) \delta$ 7.36-7.34 $(1 \mathrm{H}, \mathrm{m}), 7.23-7.19(2 \mathrm{H}, \mathrm{m}), 7.09-7.06(1 \mathrm{H}, \mathrm{m}), 6.62(1 \mathrm{H}, \mathrm{d}, \mathrm{J}=10 \mathrm{~Hz}), 5.97(1 \mathrm{H}, \mathrm{dd}$, $\mathrm{J}=9.8 \mathrm{~Hz}, 4.4 \mathrm{~Hz}), 5.03(1 \mathrm{H}, \mathrm{br} \mathrm{s}), 4.60(1 \mathrm{H}, \mathrm{br} \mathrm{s}), 3.37(1 \mathrm{H}, \mathrm{t}, \mathrm{J}=4.4 \mathrm{~Hz}), 2.61-2.56(2 \mathrm{H}$, m), 2.44-2.42 $(2 \mathrm{H}, \mathrm{m}), 1.50-137(6 \mathrm{H}, \mathrm{m}), 1.45(9 \mathrm{H}, \mathrm{s}) ;{ }^{13} \mathrm{C} \mathrm{NMR}\left(400 \mathrm{MHz}, \mathrm{CDCl}_{3}\right) \delta$ 155.32, 135.83, 132.57, 129.36, 128.23, 128.04, 127.80, 126.78, 126.39, 64.92, 50.08, 48.15, 29.82, 28.57, 26.55, 24.69. HRMS calcd for $\mathrm{C}_{20} \mathrm{H}_{28} \mathrm{~N}_{2} \mathrm{O}_{2}\left(\mathrm{M}^{+}\right): 328.2150$. Found: 328.2145 .

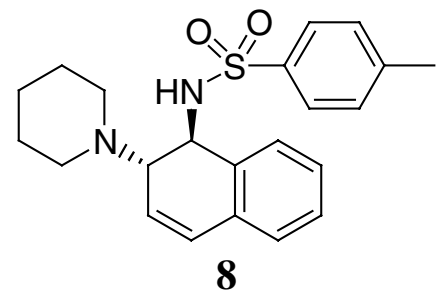

4-Methyl-N-(2-piperidin-1-yl-1,2-dihydro-naphthalen-1-yl)-benzenesulfonamide (8): To round bottomed flask was added N-Tos $4(60 \mathrm{mg}, 0.2 \mathrm{mmol})$, [Rh(COD)Cl $]_{2}(2.5 \mathrm{mg}$, $0.005 \mathrm{mmol})$, and DPPF $(5.5 \mathrm{mg}, 0.01 \mathrm{mmol})$. THF $(2 \mathrm{ml})$ was then added followed by triethylamine $(140 \mu \mathrm{l}, 1.0 \mathrm{mmol})$ and piperidine hydrochloride $(121 \mathrm{mg}, 1.0 \mathrm{mmol})$. The resulting heterogeneous mixture was heated to reflux for 14 hours. Upon completion, the reaction mixture was concentrated and chromatographed (30\% ethyl acetate:hexanes) to give 8 (72 mg, 94\%) a white solid. $\mathrm{R}_{\mathrm{f}}=0.17$ on silica gel (30\% ethyl acetate:hexanes);

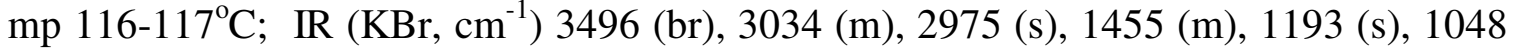
(s). ${ }^{1} \mathrm{H} \mathrm{NMR}\left(400 \mathrm{MHz}, \mathrm{CDCl}_{3}\right) \delta 7.75(2 \mathrm{H}, \mathrm{d}, J=8.2 \mathrm{~Hz}), 7.30(2 \mathrm{H}, \mathrm{d}, J=7.8 \mathrm{~Hz}), 7.21-$ $7.18(1 \mathrm{H}, \mathrm{m}), 7.10-7.05(1 \mathrm{H}, \mathrm{m}), 7.04(1 \mathrm{H}, \mathrm{d}, J=7.5 \mathrm{~Hz}), 6.94(1 \mathrm{H}, \mathrm{d}, J=7.5 \mathrm{~Hz}), 6.61$ $(1 \mathrm{H}, \mathrm{dd}, J=1.0,9.7 \mathrm{~Hz}), 5.91(1 \mathrm{H}, \mathrm{dd}, J=4.8,9.7 \mathrm{~Hz}), 4.82(1 \mathrm{H}, \mathrm{s}(\mathrm{br})), 4.53(1 \mathrm{H}, \mathrm{d}, J=$ 4.4Hz), 3.38-3.35 (1H, m), $2.44(3 \mathrm{H}, \mathrm{s}), 2.41-2.34(2 \mathrm{H}, \mathrm{m}), 2.16-2.09(2 \mathrm{H}, \mathrm{m}), 1.40-1.26$ $(6 \mathrm{H}, \mathrm{m}) ;{ }^{13} \mathrm{C}$ NMR $\left(400 \mathrm{MHz}, \mathrm{CDCl}_{3}\right) \delta 143.3,137.7,134.2,132.2,129.6,129.4,128.2$, 128.0, 127.7, 127.2, 126.6, 125.0, 64.2, 50.9, 49.6, 26.2, 24.3, 21.5. HRMS calcd for $\mathrm{C}_{22} \mathrm{H}_{26} \mathrm{~N}_{2} \mathrm{O}_{2} \mathrm{~S}\left(\mathrm{M}^{+}\right): 382.1715$. Found: 382.1713 . 


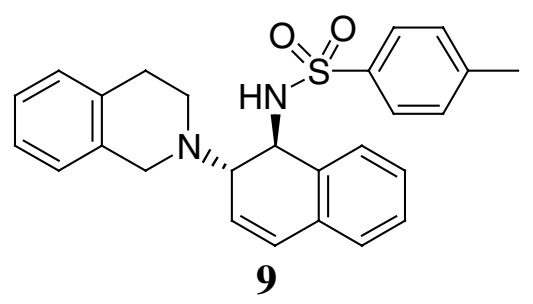

N-[2-(3,4-Dihydro-1H-isoquinolin-2-yl)-1,2-dihydro-naphthalen-1-yl]-4-methylbenzenesulfonamide (9): To round bottomed flask was added N-Tos 4 (60 mg, 0.2 $\mathrm{mmol}),[\mathrm{Rh}(\mathrm{COD}) \mathrm{Cl}]_{2}(2.5 \mathrm{mg}, 0.005 \mathrm{mmol})$, and DPPF $(5.5 \mathrm{mg}, 0.01 \mathrm{mmol})$. THF (2 $\mathrm{ml})$ was then added followed by triethylamine hydrochloride $(138 \mathrm{mg}, 1.0 \mathrm{mmol})$ and tetrahydroisoqinoline $(125 \mu \mathrm{l}, 1.0 \mathrm{mmol})$. The resulting heterogeneous mixture was heated to reflux for 15 hours. Upon completion, the reaction mixture was concentrated and chromatographed (30\% ethyl acetate:hexanes) to give $9(70 \mathrm{mg}, 81 \%)$ as a white solid. $\mathrm{R}_{\mathrm{f}}=0.22$ on silica gel (30\% ethyl acetate:hexanes); $\mathrm{mp} 142-146^{\circ} \mathrm{C} ; \mathrm{IR}\left(\mathrm{KBr}, \mathrm{cm}^{-1}\right)$ 3482 (br), 3036 (w), 2937 (s), 2853 (m), 1453 (s), 1193 (s), 1109 (s), 1046 (s) . ${ }^{1} \mathrm{H}$ NMR $\left(400 \mathrm{MHz}, \mathrm{CDCl}_{3}\right) \delta 7.73(2 \mathrm{H}, \mathrm{d}, J=8.2 \mathrm{~Hz}), 7.26-7.18(3 \mathrm{H}, \mathrm{m}), 7.12-6.98(5 \mathrm{H}, \mathrm{m}), 6.90$ $(1 \mathrm{H}, \mathrm{d}, J=8.1 \mathrm{~Hz}), 6.80(1 \mathrm{H}, \mathrm{d}, J=6.8 \mathrm{~Hz}), 6.67(1 \mathrm{H}, \mathrm{d}, J=9.7 \mathrm{~Hz}), 5.95(1 \mathrm{H}, \mathrm{dd}, J=4.7$, $9.7 \mathrm{~Hz}), 4.80(1 \mathrm{H}, \mathrm{s}), 4.62(1 \mathrm{H}, \mathrm{s}), 3.68(1 \mathrm{H}, \mathrm{AB}, \mathrm{d}, J=15.0 \mathrm{~Hz}), 3.63(1 \mathrm{H}, \mathrm{dd}, J=4.5$, $4.5 \mathrm{~Hz}), 3.40(1 \mathrm{H}, \mathrm{AB}, \mathrm{d}, J=15.0 \mathrm{~Hz}), 2.68-2.56(4 \mathrm{H}, \mathrm{m}), 2.40(3 \mathrm{H}, \mathrm{s}) ;{ }^{13} \mathrm{C}$ NMR $\left(400 \mathrm{MHz}, \mathrm{CDCl}_{3}\right) \delta 143.4,137.7,137.7,134.1,133.8,132.2,129.9,129.6,128.6,128.5$, 128.3, 127.9, 127.2, 126.8, 126.5, 125.9, 125.4, 124.6. Anal. Calcd for $\mathrm{C}_{26} \mathrm{H}_{26} \mathrm{~N}_{2} \mathrm{O}_{2} \mathrm{~S}: \mathrm{C}$, 72.53; H, 6.09; N, 6.51. Found: C, 72.56; H, 6.12; N, 6.50.

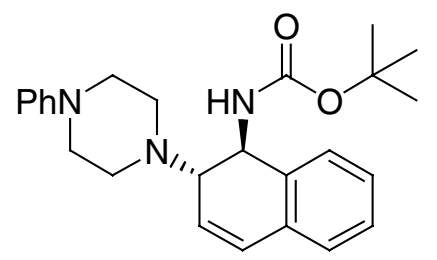

(10)

(1S, 2S)-[2-(4-Phenyl-piperazin-1-yl)-1,2-dihydro-naphthalen-1-yl]-carbamic acid tert-butyl ester (10).

Following the general procedure, $\mathbf{1 0}$ was obtained as a white solid (126mg, 76\%). The ee was determined to be $96 \%$ using HPLC analysis on a CHIRALCEL AD column, $\lambda=$ $486 \mathrm{~nm}$. Retention times in 10\% isopropanol in hexanes were 7.13min (minor) and 8.46 $\min$ (major). $\mathrm{R}_{\mathrm{f}}=0.2$ on silica gel $\left(10 \%\right.$ ethyl acetate:hexanes); $\mathrm{mp} 166-167^{\circ} \mathrm{C}\left(\mathrm{Et}_{2} \mathrm{O}\right)$; $[\alpha]^{25}=+314.8\left(\mathrm{c}=0.99, \mathrm{CCl}_{4}\right) ; \quad \mathrm{IR}\left(\mathrm{KBr}, \mathrm{cm}^{-1}\right) 3453(\mathrm{~m}), 2979(\mathrm{~m}), 2328(\mathrm{~m}), 1711(\mathrm{~s})$, 1562(m), 1483(m), 1367(m), 1169(s); ${ }^{1} \mathrm{H}$ NMR $\left(400 \mathrm{MHz}, \mathrm{CDCl}_{3}\right) \delta 7.37(1 \mathrm{H}, \mathrm{m})$, 7.25(4H, m), 7.11-7.09(1H, m), $6.86(2 \mathrm{H}, \mathrm{d}, \mathrm{J}=8.4 \mathrm{~Hz}), 6.82(1 \mathrm{H}, \mathrm{t}, \mathrm{J}=7.2 \mathrm{~Hz}), 6.66(1 \mathrm{H}, \mathrm{d}$, $\mathrm{J}=10 \mathrm{~Hz}), 6.00(1 \mathrm{H}, \mathrm{dd}, \mathrm{J}=9.6 \mathrm{~Hz}, 4.4 \mathrm{~Hz}), 5.06(1 \mathrm{H}, \mathrm{br} s), 4.60(1 \mathrm{H}, \mathrm{br} d, \mathrm{~J}=6.4 \mathrm{~Hz})$, $3.47(1 \mathrm{H}, \mathrm{t}, \mathrm{J}=4.4 \mathrm{~Hz}), 3.10(4 \mathrm{H}, \mathrm{t}, \mathrm{J}=5.2 \mathrm{~Hz}), 2.82-2.76(2 \mathrm{H}, \mathrm{m}), 2.70-2.68(2 \mathrm{H}, \mathrm{m})$, $1.44(9 \mathrm{H}, \mathrm{s}) ;{ }^{13} \mathrm{C} \mathrm{NMR}\left(400 \mathrm{MHz}, \mathrm{CDCl}_{3}\right) \delta 155.38,151.57,135.37,132.53,129.82$, $129.17,128.42,128.27,127.95,126.90,125.48,119.68,116.22,64.03,49.76,48.81$, 48.64, 29.83, 28.61. HRMS calcd for $\mathrm{C}_{25} \mathrm{H}_{31} \mathrm{~N}_{3} \mathrm{O}_{2}\left(\mathrm{M}^{+}\right)$: 405.2416. Found: 405.2411. 


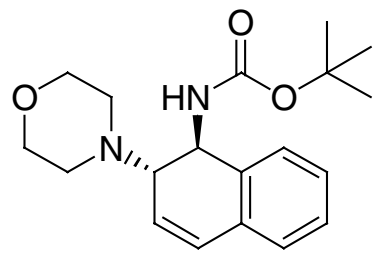

(11)

(1S, 2S)-(2-Morpholin-4-yl-1,2-dihydro-naphthalen-1-yl)-carbamic acid tert-butyl ester (11)

Following the general procedure, $\mathbf{1 1}$ was obtained as a white solid (90mg, 67\%). The ee was determined to be $80 \%$ using HPLC analysis on a CHIRALCEL AD column, $\lambda=486$ $\mathrm{nm}$. Retention times in $10 \%$ isopropanol in hexanes $(0.5 \mathrm{ml} / \mathrm{min}, 15 \mathrm{C})$ were $15.27 \mathrm{~min}$ (minor) and $16.72 \min$ (major). $\mathrm{R}_{\mathrm{f}}=0.3$ on silica gel ( $40 \%$ ethyl acetate:hexanes); $\mathrm{mp}$ $113-116^{\circ} \mathrm{C}\left(\mathrm{Et}_{2} \mathrm{O}\right) ;[\alpha]^{25}=+210.3\left(\mathrm{c}=1.04, \mathrm{CCl}_{4}\right) ; \mathrm{IR}\left(\mathrm{KBr}, \mathrm{cm}^{-1}\right) 3448(\mathrm{~m}), 2978(\mathrm{~m})$, 2281(w), 1714(s), 1550(s), 1367(w), 1250(s), 1170(s), 1005(s), 757(s); ${ }^{1} \mathrm{H}$ NMR $\left(400 \mathrm{MHz}, \mathrm{CDCl}_{3}\right) \delta$ 7.37-7.35(1H, m), 7.2-7.21(2H, m), 7.10-7.08(1H, m), 6.65(1H, d, $\mathrm{J}=9.6 \mathrm{~Hz}), 5.97(1 \mathrm{H}, \mathrm{dd}, \mathrm{J}=9.4 \mathrm{~Hz}, 4.4 \mathrm{~Hz}), 5.02(1 \mathrm{H}, \mathrm{br} \mathrm{s}), 4.59(1 \mathrm{H}, \mathrm{br} \mathrm{d}, \mathrm{J}=7.2 \mathrm{~Hz}), 3.62-$ $3.61(4 \mathrm{H}, \mathrm{m}), 3.36(1 \mathrm{H}, \mathrm{t}, \mathrm{J}=4 \mathrm{~Hz}), 2.65-2.62(2 \mathrm{H}, \mathrm{m}), 2.54-2.52(2 \mathrm{H}, \mathrm{m}), 1.45(9 \mathrm{H}, \mathrm{s}) ;{ }^{13} \mathrm{C}$ NMR $\left(400 \mathrm{MHz}, \mathrm{CDCl}_{3}\right) \delta 155.37,135.27,132.50,129.88,128.44,128.29,127.89$, 126.90, 125.29, 67.52, 64.35, 49.33, 48.64, 28.58. HRMS calcd for $\mathrm{C}_{19} \mathrm{H}_{26} \mathrm{~N}_{2} \mathrm{O}_{3}\left(\mathrm{M}^{+}\right)$: 330.1943. Found: 330.1945.

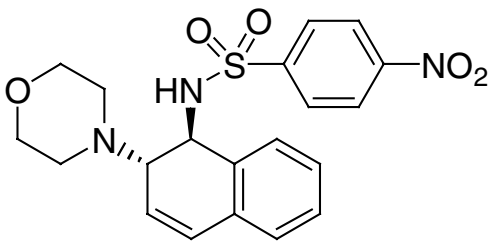

(12)

N-(2-Morpholin-4-yl-1,2-dihydro-naphthalen-1-yl)-4-nitro-benzenesulfonamide (12): To round bottomed flask was added N-Nos $4(5 \mathrm{~g}, 15.2 \mathrm{mmol}),[\mathrm{Rh}(\mathrm{COD}) \mathrm{Cl}]_{2}(1 \mathrm{~mol} \%)$, and DPPF (3 mol\%). THF (60 ml) was then added and the mixture was stirred for approximately 5 minutes. It was then heated to reflux. At first sign of reflux, $\mathrm{NH}_{4} \mathrm{I}(3.3$ $\mathrm{g}, 22.8 \mathrm{mmol}$ ) was added followed by morpholine $(4 \mathrm{~mL}, 46 \mathrm{mmol})$. The resulting heterogeneous mixture was heated to reflux for 15 hours. Upon completion, the reaction mixture was poured into an aqueous sodium bicarbonate solution and extracted with ethyl acetate. The organic fractions were combined and concentrated. The resulting crude residue was purified by chromatography (30\% ethyl acetate:hexanes) to give $\mathbf{1 2}$ (5.8 g, 92\%) an off white solid. $\mathrm{R}_{\mathrm{f}}=0.33$ on silica gel (40\% ethyl acetate:hexanes); mp 152$155^{\circ} \mathrm{C}$; IR (KBr, cm $\left.{ }^{-1}\right) 3482$ (br), 3036 (w), 2937 (s), 2853 (m), 1453 (s), 1193 (s), 1109 (s), $1046(\mathrm{~s}) .{ }^{1} \mathrm{H}$ NMR $\left(400 \mathrm{MHz}, \mathrm{CDCl}_{3}\right) \delta 8.31(2 \mathrm{H}, \mathrm{d}, J=8.8 \mathrm{~Hz}), 7.98(2 \mathrm{H}, \mathrm{d}, J=8.8$ $\mathrm{Hz}), 7.23-7.15(1 \mathrm{H}, \mathrm{m}), 7.08-6.99(2 \mathrm{H}, \mathrm{m}), 7.75(1 \mathrm{H}, \mathrm{d}, J=7.3 \mathrm{~Hz}), 7.66(1 \mathrm{H}, \mathrm{d}, J=9.8$ $\mathrm{Hz}), 5.90(1 \mathrm{H}, \mathrm{dd}, J=5.1,9.8 \mathrm{~Hz}), 5.18(1 \mathrm{H}, \mathrm{s}), 4.65(1 \mathrm{H}, \mathrm{s}), 3.54-3.44(4 \mathrm{H}, \mathrm{m}), 3.42-$ $3.37(1 \mathrm{H}, \mathrm{m}), 2.54-2.44(2 \mathrm{H}, \mathrm{m}), 2.34-2.24(2 \mathrm{H}, \mathrm{m}) ;{ }^{13} \mathrm{C} \mathrm{NMR}\left(400 \mathrm{MHz}, \mathrm{CDCl}_{3}\right) \delta$ 
149.6, 146.8, 132.5, 131.9, 129.9, 129.8, 128.2, 128.0, 127.5, 127.0, 124.1, 123.7, 67.1, 63.9, 51.9, 49.0. HRMS calcd for $\mathrm{C}_{20} \mathrm{H}_{21} \mathrm{~N}_{3} \mathrm{O}_{5} \mathrm{~S}\left(\mathrm{M}^{+}\right)$: 415.1202. Found: 415.1203.

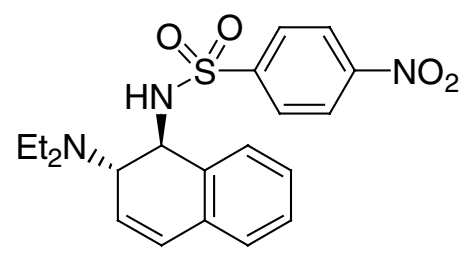

(13)

N-(2-Diethylamino-1,2-dihydro-naphthalen-1-yl)-4-nitro-benzenesulfonamide (13): To round bottomed flask was added N-Nos 4 (5 g, $15.2 \mathrm{mmol}),[\mathrm{Rh}(\mathrm{COD}) \mathrm{Cl}]_{2}(1 \mathrm{~mol} \%)$, and DPPF (3 mol\%). THF $(60 \mathrm{ml})$ was then added and the mixture was stirred for approximately 5 minutes. It was then heated to reflux. At first sign of reflux, $\mathrm{NH}_{4} \mathrm{I}(3.3$ $\mathrm{g}, 22.8 \mathrm{mmol})$ was added followed by diethylamine $(3.4 \mathrm{~mL}, 46 \mathrm{mmol})$. The resulting heterogeneous mixture was heated to reflux for 15 hours. Upon completion, the reaction mixture was poured into an aqueous sodium bicarbonate solution and extracted with ethyl acetate. The organic fractions were combined and concentrated. The resulting crude residue was purified by chromatography (30\% ethyl acetate:hexanes) to give 13 (5.5 g, $91 \%)$ an off white solid. $\mathrm{R}_{\mathrm{f}}=0.22$ on silica gel ( $40 \%$ ethyl acetate:hexanes); $\mathrm{mp} 92-95^{\circ} \mathrm{C}$; IR (KBr, cm $\left.{ }^{-1}\right) 3482$ (br), 3036 (w), 2937 (s), 2853 (m), 1453 (s), 1193 (s), 1109 (s), 1046 (s). ${ }^{1} \mathrm{H} \mathrm{NMR}\left(400 \mathrm{MHz}, \mathrm{CDCl}_{3}\right) \delta 8.32(2 \mathrm{H}, \mathrm{d}, J=8.8 \mathrm{~Hz}), 7.98(2 \mathrm{H}, \mathrm{d}, J=8.8 \mathrm{~Hz}), 7.24-$ $7.18(1 \mathrm{H}, \mathrm{m}), 7.08-7.00(2 \mathrm{H}, \mathrm{m}), 6.75(1 \mathrm{H}, \mathrm{d}, J=7.5 \mathrm{~Hz}), 6.67(1 \mathrm{H}, \mathrm{d}, J=9.8 \mathrm{~Hz}), 5.90$ $(1 \mathrm{H}, \mathrm{dd}, J=5.1,9.8 \mathrm{~Hz}), 5.88(1 \mathrm{H}, \mathrm{s}), 4.66(1 \mathrm{H}, \mathrm{s}), 3.58-3.44(4 \mathrm{H}, \mathrm{m}), 3.43-3.38(1 \mathrm{H}, \mathrm{m})$, 2.54-2.45 (2H, m), 2.34-2.28 (2H, m); ${ }^{13} \mathrm{C} \mathrm{NMR}\left(400 \mathrm{MHz}, \mathrm{CDCl}_{3}\right) \delta 149.7,146.8$, 132.6, 131.9, 129.9, 128.8, 128.3, 128.1, 127.6, 127.1, 124.1, 123.7, 67.1, 63.9, 51.9, 49.0. HRMS calcd for $\mathrm{C}_{20} \mathrm{H}_{23} \mathrm{~N}_{3} \mathrm{O}_{4} \mathrm{~S}\left(\mathrm{M}^{+}\right)$: 401.1409. Found: 401.1407.<smiles>CC(C)(C)OC(=O)N[C@H]1c2ccccc2C=C[C@H]1N(Cc1ccccc1)C(C)(C)C</smiles>

(14)

(1S, 2S)-(2-Dibenzylamino-1,2-dihydro-naphthalen-1-yl)-carbamic acid tert-butyl ester (14).

Following the general procedure, 14 was obtained as an oil (140mg, 78\%). The ee was determined to be $89 \%$ using HPLC analysis on a CHIRALCEL OD column, $\lambda=486 \mathrm{~nm}$. Retention times in $1 \%$ isopropanol in hexanes were 5.76 min (major) and $6.29 \mathrm{~min}$. $\mathrm{R}_{\mathrm{f}}=$ 0.4 on silica gel $(10 \%$ ethyl acetate:hexanes $) ;[\alpha]^{25}=+96.1\left(\mathrm{c}=1.06, \mathrm{CCl}_{4}\right) ; \mathrm{IR}(\mathrm{KBr}$, $\left.\mathrm{cm}^{-1}\right)$ 3447(m), 2280(w), 2000(w), 1954(w), 1712(s), 1549(s), 1366(m), 1251(s), 1170(s), 1005(s), 801(s); ${ }^{1} \mathrm{H}$ NMR $\left(400 \mathrm{MHz}, \mathrm{CDCl}_{3}\right) \delta 7.37-7.32(5 \mathrm{H}, \mathrm{m}), 7.27-7.24(4 \mathrm{H}, \mathrm{m})$, 7.20-7.17(4H, m), 7.03-7.00(1H, m), 6.57(1H, dd, J=9.8Hz, $1.6 \mathrm{~Hz}), 6.03(1 \mathrm{H}, \mathrm{dd}$, $\mathrm{J}=9.8 \mathrm{~Hz}, 3.2 \mathrm{~Hz}), 5.25(1 \mathrm{H}, \mathrm{t}, \mathrm{J}=5.6 \mathrm{~Hz}), 4.47(1 \mathrm{H}, \mathrm{br} \mathrm{d}, \mathrm{J}=8.8 \mathrm{~Hz}), 3.74(2 \mathrm{H}, \mathrm{d}, \mathrm{J}=13.6 \mathrm{~Hz})$, $3.54(2 \mathrm{H}, \mathrm{d}, \mathrm{J}=13.6 \mathrm{~Hz}), 3.47-3.45(1 \mathrm{H}, \mathrm{m}), 1.52(9 \mathrm{H}, \mathrm{s}) ;{ }^{13} \mathrm{C} \mathrm{NMR}\left(400 \mathrm{MHz}, \mathrm{CDCl}_{3}\right) \delta$ 
$155.67,140.06,135.75,132.58,129.50,129.19,129.10,128.25,128.16,127.85,127.35$, $127.02,126.91,126.67,79.47,59.46,54.04,49.38,28.64,28.49$. HRMS calcd for $\mathrm{C}_{29} \mathrm{H}_{32} \mathrm{~N}_{2} \mathrm{O}_{2}\left(\mathrm{M}^{+}\right): 440.2463$. Found: 440.2455 .

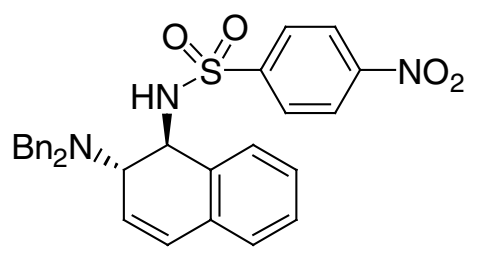

15

N-(2-Dibenzylamino-1,2-dihydro-naphthalen-1-yl)-4-nitro-benzenesulfonamide (15): To round bottomed flask was added N-Nos $4(500 \mathrm{mg}, 1.52 \mathrm{mmol}),[\mathrm{Rh}(\mathrm{COD}) \mathrm{Cl}]_{2}(1$ mol\%), and DPPF (3 mol\%). THF ( $8 \mathrm{ml}$ ) was then added and the mixture was stirred for approximately 5 minutes. It was then heated to reflux. At first sign of reflux, $\mathrm{NH}_{4} \mathrm{I}$ (330 $\mathrm{mg}, 2.28 \mathrm{mmol})$ was added followed by dibenzylamine $(900 \mathrm{~mL}, 4.6 \mathrm{mmol})$. The resulting heterogeneous mixture was heated to reflux for 15 hours. Upon completion, the reaction mixture was poured into an aqueous sodium bicarbonate solution and extracted with ethyl acetate. The organic fractions were combined and concentrated. The resulting crude residue was purified by chromatography (30\% ethyl acetate:hexanes) to give $\mathbf{1 5}$ (710 mg, 89\%) an off white solid. $\mathrm{R}_{\mathrm{f}}=0.16$ on silica gel (15\% ethyl acetate:hexanes); $\mathrm{mp}$ 192-194 ${ }^{\circ} \mathrm{C}$; IR (KBr, cm $\left.{ }^{-1}\right) 3482$ (br), 3036 (w), 2937 (s), 2853 (m), 1453 (s), 1363 (s), 1109 (s), 1046 (s). ${ }^{1} \mathrm{H}$ NMR $\left(400 \mathrm{MHz}, \mathrm{CDCl}_{3}\right) \delta 8.08(2 \mathrm{H}, \mathrm{d}, J=8.8 \mathrm{~Hz}), 7.67(2 \mathrm{H}, \mathrm{d}, J=$ $8.8 \mathrm{~Hz}), 7.25-7.14(13 \mathrm{H}, \mathrm{m}), 7.06-7.00(2 \mathrm{H}, \mathrm{m}), 6.92(1 \mathrm{H}, \mathrm{d}, J=7.2 \mathrm{~Hz}), 6.68(1 \mathrm{H}, \mathrm{d}, J=$ $9.7 \mathrm{~Hz}), 5.90(1 \mathrm{H}, \mathrm{dd}, J=5.1,9.7 \mathrm{~Hz}), 4.94-4.88(1 \mathrm{H}, \mathrm{m}), 4.81-4.75(1 \mathrm{H}, \mathrm{m}), 3.51-3.47$ $(1 \mathrm{H}, \mathrm{m}), 3.44(4 \mathrm{H}, \mathrm{d}, J=4.6 \mathrm{~Hz}) ;{ }^{13} \mathrm{C}$ NMR $\left(400 \mathrm{MHz}, \mathrm{CDCl}_{3}\right) \delta 149.4,147.0,139.4$, 132.7, 132.0, 129.8, 128.8, 128.3, 128.1, 128.0, 127.6, 127.1, 126.9, 124.9, 123.9, 57.7, 53.3, 52.7. HRMS calcd for $\mathrm{C}_{30} \mathrm{H}_{27} \mathrm{~N}_{3} \mathrm{O}_{4} \mathrm{~S}\left(\mathrm{M}^{+}\right)$: 525.1722. Found: 525.1725.

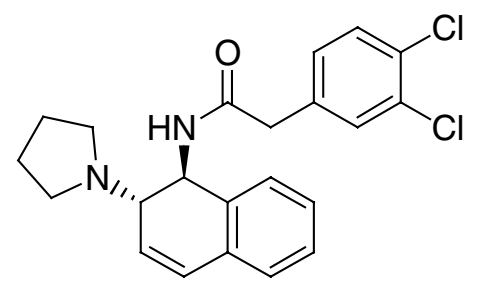

(1S, 2S)-2-(3,4-Dichloro-phenyl)-N-(2-pyrrolidin-1-yl-1,2-dihydro-naphthalen-1-yl)acetamide: To round bottomed flask was added 2-(3,4-Dichloro-phenyl)-1-(1,4 dihydro-1,4-epizano-naphthalen-9yl)-ethanone $(66 \mathrm{mg}, 0.2 \mathrm{mmol}),[\mathrm{Rh}(\mathrm{COD}) \mathrm{Cl}]_{2}(2.5$ $\mathrm{mg}, 0.005 \mathrm{mmol})$, and $\mathrm{C}_{2}$-Ferriphos $(5.5 \mathrm{mg}, 0.01 \mathrm{mmol})$. THF $(2 \mathrm{ml})$ was then added followed by triethylamine hydrochloride $(138 \mathrm{mg}, 1.0 \mathrm{mmol})$ and pyrrolidine $(125 \mu \mathrm{l}, 1.0$ mmol). The resulting heterogeneous mixture was heated to reflux for 15 hours. Upon completion, the reaction mixture was concentrated and chromatographed (50\% ethyl acetate:hexanes with $\left.5 \% \mathrm{Et}_{3} \mathrm{~N}\right)$ to give the product as a tan solid $(78 \mathrm{mg}, 98 \%)$. The ee was determined to be $99 \%$ using HPLC analysis on a CHIRALCEL OD column, $\lambda=486$ 
$\mathrm{nm}$. Retention times in $10 \%$ isopropanol in hexanes were $13.8 \mathrm{~min}$ (major) and 18.68min. $\quad \mathrm{R}_{\mathrm{f}}=0.1$ on silica gel $\left(50 \%\right.$ ethyl acetate:hexanes); mp $78-84^{\circ} \mathrm{C} ;[\alpha]^{25}{ }_{\mathrm{D}}=$ +59.4 (c= 0.98, $\left.\mathrm{CHCl}_{3}\right)$; IR $\left(\mathrm{KBr}, \mathrm{cm}^{-1}\right)$ 3683(m), 3014(s), 2400(s), 2246(w), 1968(w), 1883(w), 1670(s), 1518(s), 1423(s), 1220(s), 1033(s), 929(s), 805(s). ${ }^{1} \mathrm{H}$ NMR (400MHz, $\left.\mathrm{CDCl}_{3}\right) \delta$ 7.36-7.31(1H, m), 7.26-7.19(4H, m), 7.11-7.04(2H, m), 6.61(1H, d, J=12.8Hz), $5.97(1 \mathrm{H}, \mathrm{dd}, \mathrm{J}=13.2 \mathrm{~Hz}, 6.4 \mathrm{~Hz}), 5.76(1 \mathrm{H}, \mathrm{d}, \mathrm{J}=10.8 \mathrm{~Hz}), 5.28-5.23(1 \mathrm{H}, \mathrm{m}), 3.41(2 \mathrm{H}, \mathrm{s})$, $3.31(1 \mathrm{H}, \mathrm{t}, \mathrm{J}=6 \mathrm{~Hz}), 2.67-2.55(4 \mathrm{H}, \mathrm{m}), 2.02(1 \mathrm{H}$, br s$), 1.69-1.65(4 \mathrm{H}, \mathrm{m}) ;{ }^{13} \mathrm{C} \mathrm{NMR}$ $\left(400 \mathrm{MHz}, \mathrm{CDCl}_{3}\right) \delta 168.95,135.02,134.03,132.52,132.22,131.18,131.06,130.45$, $129.24,128.52,128.30,128.20,128.03,126.82,126.13,60.54,49.82,49.59,42.41$, 23.46. HRMS calcd for $\mathrm{C}_{18} \mathrm{H}_{14} \mathrm{NOCl}_{2}\left[\left(\mathrm{M}-\mathrm{C}_{4} \mathrm{H}{ }_{9} \mathrm{~N}\right)^{+}\right]$: 329.0374 . Found: 329.0366

\section{Preparation of Analgesic Compound 3.}

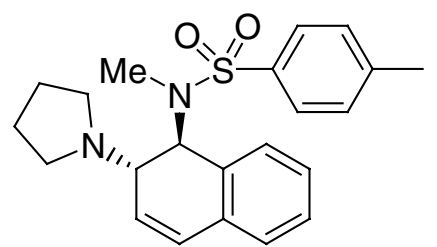

(+/-) 16a

4,N-Dimethyl-N-(2-pyrrolidin-1-yl-1,2-dihydro-naphthalen-1-yl)-

benzenesulfonamide [(+/-) 16a]: To a round bottomed flask was added (+/-) 5a (100 mg, $0.27 \mathrm{mmol})$ and potassium carbonate $(112 \mathrm{mg}, 0.81 \mathrm{mmol})$. Acetone $(3 \mathrm{ml})$ was then added followed by iodomethane $(18 \mu \mathrm{l}, 0.28 \mathrm{mmol})$. The mixture was stirred at room temperature for 4 hours then quenched with water. Extraction with ethylacetate, combining of the organic fractions and concentration gave a light yellow solid. Chromatography (30\% ethyl acetate:hexanes) gave pure (+/-) 16a (101 mg, 98\%) a white crystalline solid. $\mathrm{R}_{\mathrm{f}}=0.18$ on silica gel (30\% ethyl acetate:hexanes); mp $109-111^{\circ} \mathrm{C}$; IR (KBr, cm $\left.{ }^{-1}\right) 3386$ (br), 3016 (m), 2984 (s), 1497 (s), 1343 (s), 1117 (m), 1048 (s). ${ }^{1} \mathrm{H}$ NMR $\left(400 \mathrm{MHz}, \mathrm{CDCl}_{3}\right) \delta 7.86(2 \mathrm{H}, \mathrm{d}, J=8.0 \mathrm{~Hz}), 7.31(2 \mathrm{H}, \mathrm{d}, J=8.0 \mathrm{~Hz}), 7.26-7.12(3 \mathrm{H}$, $\mathrm{m}), 7.06(1 \mathrm{H}, \mathrm{d}, J=6.9 \mathrm{~Hz}), 6.58(1 \mathrm{H}, \mathrm{d}, J=9.7 \mathrm{~Hz}), 5.95(1 \mathrm{H}, \mathrm{dd}, J=4.6,9.9 \mathrm{~Hz}), 5.35$ $(1 \mathrm{H}, \mathrm{d}, J=4.5 \mathrm{~Hz}), 3.42(1 \mathrm{H}, \mathrm{dd}, J=4.5,4.5 \mathrm{~Hz}), 2.62-2.48(4 \mathrm{H}, \mathrm{m}), 2.50(3 \mathrm{H}, \mathrm{s}), 2.45$ $(3 \mathrm{H}, \mathrm{s}), 1.70-1.63(4 \mathrm{H}, \mathrm{m}) ;{ }^{13} \mathrm{C} \mathrm{NMR}\left(400 \mathrm{MHz}, \mathrm{CDCl}_{3}\right) \delta 143.0,137.7,133.6,132.1$, 129.5, 129.1, 128.9, 128.2, 128.1, 127.4, 126.5, 125.9, 58.2, 56.5, 48.6, 29.6, 23.5, 21.5. Anal. Calcd for $\mathrm{C}_{22} \mathrm{H}_{26} \mathrm{~N}_{2} \mathrm{O}_{2} \mathrm{~S}$ : C, 69.08; H, 6.85; N, 7.32. Found: C, 69.14; H, 6.91; N, 7.30 .

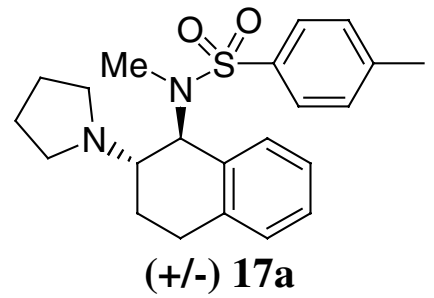

4,N-Dimethyl-N-(2-pyrrolidin-1-yl-1,2,3,4-tetrahydro-naphthalen-1-yl)benzenesulfonamide [(+/-) 17a]: To a round bottomed flask was added (+/-) 16a (100 
$\mathrm{mg}, 0.26 \mathrm{mmol})$, ethylacetate $(2 \mathrm{ml})$ and palladium on carbon $(5 \mathrm{mg})$. Hydrogen was added over this heterogeneous mixture via balloon for 15 hours. Upon completion, the mixture was filtred through celite and concentrated to give (+/-) $\mathbf{1 7 a}$ as a white solid. Crude ${ }^{1} \mathrm{H}$ NMR showed that this crude product was $>95 \%$ pure. Further purification could be obtained by chromatography (30\% ethyl acetate:hexanes) giving pure (+/-) 17a (98 mg, 98\%). $\mathrm{R}_{\mathrm{f}}=0.15$ on silica gel (30\% ethyl acetate:hexanes); mp $109-110^{\circ} \mathrm{C}$; IR $\left(\mathrm{KBr}, \mathrm{cm}^{-1}\right) 3384$ (br), 3009 (m), 2978 (s), 1338 (s), 1115 (m), 1052 (s). ${ }^{1} \mathrm{H}$ NMR $\left(400 \mathrm{MHz}, \mathrm{CDCl}_{3}\right) \delta 7.99(2 \mathrm{H}, \mathrm{d}, J=8.1 \mathrm{~Hz}), 7.30(2 \mathrm{H}, \mathrm{d}, J=8.3 \mathrm{~Hz}), 7.15-7.02(4 \mathrm{H}, \mathrm{m})$, $5.29(1 \mathrm{H}, \mathrm{d}, J=8.1 \mathrm{~Hz}), 3.03-2.67(5 \mathrm{H}, \mathrm{m}), 2.65-2.52(2 \mathrm{H}, \mathrm{m}), 2.44(3 \mathrm{H}, \mathrm{s}), 2.43(3 \mathrm{H}, \mathrm{s})$, 2.05-1.96 (1H, m), 1.90-1.80 (1H, m), 1.72-1.64 (4H, m); ${ }^{13} \mathrm{C} \mathrm{NMR}\left(400 \mathrm{MHz}, \mathrm{CDCl}_{3}\right) \delta$ $142.8,138.5,137.5,133.9,129.2,128.5,127.7,127.1,126.4,60.0,59.3,48.7,30.3,27.9$, 23.6, 21.5, 21.4. Anal. Calcd for $\mathrm{C}_{22} \mathrm{H}_{28} \mathrm{~N}_{2} \mathrm{O}_{2} \mathrm{~S}: \mathrm{C}, 68.72 ; \mathrm{H}, 7.34 ; \mathrm{N}, 7.29$. Found: C, $68.79 ; \mathrm{H}, 7.37 ; \mathrm{N}, 7.22$.

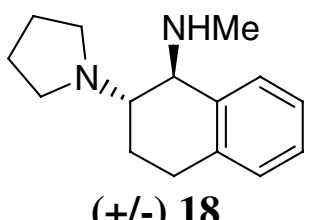

Methyl-(2-pyrrolidin-1-yl-1,2,3,4-tetrahydro-naphthalen-1-yl)-amine [(+/-) 18]: To a quartz tube was added (+/-) 17a $(80 \mathrm{mg}, 0.2 \mathrm{mmol}), 1,4$-dimethoxybenzene $(110 \mathrm{mg}, 0.8$ mmol) and sodium borohydride $(76 \mathrm{mg}, 2.0 \mathrm{mmol})$ followed by $90 \%$ aqueous ethanol solution $(3 \mathrm{ml})$. The mixture was irradiated at $254 \mathrm{~nm}$ in a rayonet reactor for 2.5 hours. The crude mixture was concentrated azeotropically with ethanol and then chromatographed (90\% acetone, $9 \% \mathrm{MeOH}, 1 \%$ triethylamine) to give (+/-) $\mathbf{1 8}(42 \mathrm{mg}$, $91 \%)$. Spectral data was identical to the literature data. ${ }^{5}$ This compound was then converted to the analgesic compound $\mathbf{3}$ by the literature procedure.

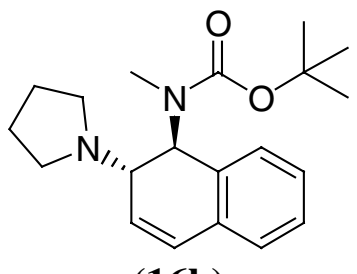

(16b)

(1S, 2S)-Methyl-(2-pyrrolidin-1-yl-1,2-dihydro-naphthalen-1-yl)-carbamic acid tertbutyl ester $[(+)-16 b]$.

$\mathrm{KH}(35 \%$ dispersion in mineral oil, 1.0g, 2 eq.) was washed three times with pentane, dried under a stream of nitrogen, and suspended in $\mathrm{THF}(20 \mathrm{ml})$. A solution of (+)-5b $(1.4 \mathrm{~g}, 4.45 \mathrm{mmol})$ in THF $(10 \mathrm{ml})$ was added dropwise via cannula. After 15 minutes at room temperature, iodomethane $(292 \mu \mathrm{l}, 4.68 \mathrm{mmol})$ is added dropwise and the resulting solution is stirred at room temperature for 1 hour. The mixture was quenched by dropwise addition of water $(25 \mathrm{ml})$ and then extracted with $\mathrm{CH}_{2} \mathrm{Cl}_{2}(3 \times 30 \mathrm{ml})$. The

${ }^{5}$ Freeman, J.P.; Michalson, E.T.; D’Andrea, S.V.; Baczynskyj, L.; Von Voigtlander, P.F.; Lahti, R.A.; Smith, M.W.; Lawson, C.F.; Scahill, T.A.; Mizsak, S.A.; Szmuszkovicz, J. J. Med. Chem 1991, 34, 1891. 
organic layers are combined, dried over $\mathrm{Na}_{2} \mathrm{SO}_{4}$, filtered, and concentrated under reduced pressure. Flash chromatography (30\% EtOAc/hexanes $\rightarrow 50 \%$ EtOAc/hexanes) provides the product as an oil $(1.38 \mathrm{~g}, 95 \%) . \mathrm{R}_{\mathrm{f}}=0.29$ on silica gel (40 \% ethyl acetate:hexanes);

$[\alpha]_{\mathrm{D}}^{25}=+17.90\left(\mathrm{c}=0.98, \mathrm{CCl}_{4}\right) ; \mathrm{R}_{\mathrm{f}}=$ on silica (\% ethyl acetate:hexanes). IR $\left(\mathrm{KBr}, \mathrm{cm}^{-1}\right)$ 3683(m), 1964(w), 1929(w), 1887(w), 3448(m), 1677(s), 1518(s), 1426(m), 1228(s), 1046(s), 928(s), 796(s); ${ }^{1} \mathrm{H}$ NMR $\left(400 \mathrm{MHz}, \mathrm{CDCl}_{3}\right)$ corresponding to a $1: 1$ mixture of rotamers : $\delta$ 7.19-7.17(3H, m), 7.07(1H, t, J=4.4Hz), 6.53(1H, t, J=7.6Hz), 6.05(1H, dd, $\mathrm{J}=9.8 \mathrm{~Hz}, 4 \mathrm{~Hz}), 5.65(0.5 \mathrm{H}, \mathrm{d}, \mathrm{J}=7.2 \mathrm{~Hz}), 5.46(0.5 \mathrm{H}, \mathrm{d}, \mathrm{J}=8.4 \mathrm{~Hz}), 3.69(0.5 \mathrm{H}, \mathrm{d}, \mathrm{J}=7.6 \mathrm{~Hz})$, 3.59(0.5H, s), 2.73-2.65(4H, m), 2.65(1.5H, s), 2.56(1.5H, s), 1.75-.173(4H, m), 1.49(9H, $\mathrm{s}) ;{ }^{13} \mathrm{C} \mathrm{NMR}\left(400 \mathrm{MHz}, \mathrm{CDCl}_{3}\right.$ ) corresponding to a $1: 1$ mixture of rotamers: $\delta 155.97$, 134.32 , 133.91, 133.78, 128.74, 128.04, 127.92, 127.81, 127.67, 127.56, 126.85, 126.68, 126.57, 79.88, 79.51, 58.83, 55.95, 54.12, 48.88, 29.61, 29.34, 28.63, 28.55, 23.76. HRMS calcd for $\mathrm{C}_{20} \mathrm{H}_{27} \mathrm{~N}_{2} \mathrm{O}_{2}\left(\mathrm{M}^{+}\right)$: 327.2072. Found: 327.2080.

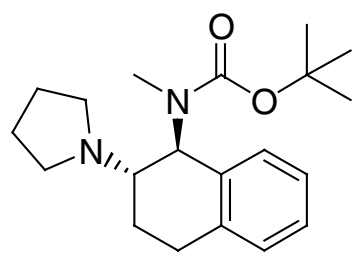

(17b)

(1S, 2S)-Methyl-(2-pyrrolidin-1-yl-1,2,3,4-tetrahydro-naphthalen-1-yl)-carbamic acid tert-butyl ester $[(+)-17 \mathrm{~b}]$.

To a mixture of $(+)-\mathbf{1 6 b}(1.2 \mathrm{~g}, 3.65 \mathrm{mmol})$ and hydrazine monohydrate $(7.1 \mathrm{ml}$, $146.32 \mathrm{mmol})$ in $95 \% \mathrm{EtOH}: \mathrm{THF}(30 \mathrm{ml}: 10 \mathrm{ml})$ at $0^{\circ} \mathrm{C}$ was added slowly a solution of $\mathrm{NaIO}_{4}(7.8 \mathrm{~g}, 36.58 \mathrm{mmol})$ in $\mathrm{H}_{2} \mathrm{O}(10 \mathrm{ml})$. The resulting slurry was heated with stirring at $70^{\circ} \mathrm{C}$ for 4 hours (with reflux condenser left open to the atmosphere), over which time the mixture turned to a clear, homogeneous solution. The solution was cooled to room temperature, diluted with $\mathrm{CH}_{2} \mathrm{Cl}_{2}$, and washed with $1 \mathrm{~N} \mathrm{NaOH}, \mathrm{H}_{2} \mathrm{O}$, and brine. The organic layer was dried $\left(\mathrm{Na}_{2} \mathrm{SO}_{4}\right)$, filtered, and concentrated under reduced pressure to afford (+)-17b as an oil $(1.1 \mathrm{~g}, 93 \%) . \mathrm{R}_{\mathrm{f}}=0.29$ on silica gel (40\% ethyl acetate:hexanes); $[\alpha]^{25}{ }_{\mathrm{D}}=+23.3\left(\mathrm{c}=1.03, \mathrm{CCl}_{4}\right) ; \mathrm{IR}\left(\mathrm{KBr}, \mathrm{cm}^{-1}\right) 2294(\mathrm{~m}), 1717(\mathrm{~s}), 1690(\mathrm{~s}), 1549(\mathrm{~s})$, 1438(w), 1365(w), 1253(s), 1217(s), 1136(s), 1005(s), 764(s); ${ }^{1}$ H NMR (400MHz, $\left.\mathrm{CDCl}_{3}\right)$ corresponding to a $1: 1$ mixture of rotamers: $\delta 7.15-7.07(4 \mathrm{H}, \mathrm{m}), 5.48(0.5 \mathrm{H}, \mathrm{d}$, $\mathrm{J}=9.2 \mathrm{~Hz}), 5.29-5.26(0.5 \mathrm{H}, \mathrm{m}), 3.02-2.76(5 \mathrm{H}, \mathrm{m}), 2.65-2.63(2 \mathrm{H}, \mathrm{m}), 2.58(1.5 \mathrm{H}, \mathrm{s})$, $2.51(1.5 \mathrm{H}, \mathrm{s}), 2.10-2.05(1 \mathrm{H}, \mathrm{m}), 1.85-1.72(5 \mathrm{H}, \mathrm{m}), 1.51(4.5 \mathrm{H}, \mathrm{s}), 1.49(4.5 \mathrm{H}, \mathrm{s}) ;{ }^{13} \mathrm{C}$ NMR $\left(400 \mathrm{MHz}, \mathrm{CDCl}_{3}\right)$ corresponding to a $1: 1$ mixture of rotamers: $\delta 156.98,156.74$, 138.03, 137.96, 136.41, 136.12, 128.61, 128.57, 128.07, 127.78, 126.86, 126.76, 126.41, $126.27,79.68,79.28,58.62,57.34,48.75,48.58,30.03,29.09,28.97,28.70,28.64,25.44$, 23.86, 23.74, 23.28, 23.15. HRMS calcd for $\mathrm{C}_{20} \mathrm{H}_{30} \mathrm{~N}_{2} \mathrm{O}_{2}\left(\mathrm{M}^{+}\right)$: 330.2307. Found: 330.2316 . 


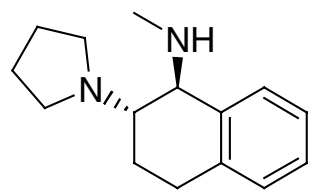

(18)

(1S, 2S)-Methyl-(2-pyrrolidin-1-yl-1,2,3,4-tetrahydro-naphthalen-1-yl)-amine [(+)18 .

In a $25 \mathrm{ml}$ round bottom flask was added (+)-17b (300mg, $0.91 \mathrm{mmol}), \mathrm{CH}_{2} \mathrm{Cl}_{2}$, and dropwise additon of trifluoroacetic acid $(1 \mathrm{ml})$. The solution is stirred at room temperature for 45 minutes. $10 \mathrm{ml}$ of $\mathrm{KOH}(10 \%$ aqueous solution) was added and the mixture was washed with $\mathrm{CH}_{2} \mathrm{Cl}_{2}(3 \times 20 \mathrm{ml})$. The organic layers are combined, dried over $\mathrm{Na}_{2} \mathrm{SO}_{4}$, filtered, and concentrated under reduced pressure to yield the product as an oil $(198 \mathrm{mg}, 95 \%) . \mathrm{R}_{\mathrm{f}}=0.1$ on silica gel $\left(100 \%\right.$ ethyl acetate); $\mathrm{mp}{ }^{\circ} \mathrm{C}\left(\mathrm{Et}_{2} \mathrm{O}\right) ;[\alpha]^{25}=$ $+65.0\left(\mathrm{c}=1.6, \mathrm{CCl}_{4}\right) ; \mathrm{IR}\left(\mathrm{KBr}, \mathrm{cm}^{-1}\right) 3459(\mathrm{~s}), 2305(\mathrm{~m}), 1550(\mathrm{~s}), 1422(\mathrm{~m}), 1264(\mathrm{~s})$, $1122(\mathrm{~m}), 1003(\mathrm{~m}), 895(\mathrm{w}) ;{ }^{1} \mathrm{H}$ NMR $\left(400 \mathrm{MHz}, \mathrm{CDCl}_{3}\right) \delta$ 7.28-7.24(1H, m), 7.157.05(3H, m), 3.66(1H, d, J=4Hz), 2.91-2.83(1H, m), 2.69-2.59(6H, m), 2.46(3H, s), 2.10$2.02(1 \mathrm{H}, \mathrm{m}), 1.88-1.81(1 \mathrm{H}, \mathrm{m}), 1.73-1.70(4 \mathrm{H}, \mathrm{m}), 1.54(1 \mathrm{H}, \mathrm{br} \mathrm{s}) ;{ }^{13} \mathrm{C} \mathrm{NMR}(400 \mathrm{MHz}$, $\left.\mathrm{CDCl}_{3}\right) \delta 137.73,137.62,129.57,128.73,126.63,125.70,61.65,60.87,51.15,34.65$, 26.02, 23.50, 22.14. HRMS calcd for $\mathrm{C}_{15} \mathrm{H}_{22} \mathrm{~N}_{2}\left(\mathrm{M}^{+}\right)$: 230.1782. Found: 230.1792 .

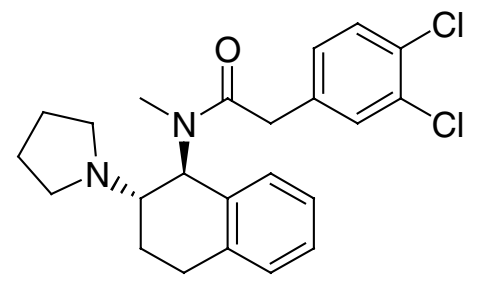

(3)

(1S, 2S)-2-(3,4-Dichloro-phenyl)- $N$-methyl- $N$-(2-pyrrolidin-1-yl-1,2,3,4-tetrahydonaphthalen-1-yl)-acetamide (3) : In a 50ml round bottom flask was added (+)-21 (200mg, 0.86mmol), $\mathrm{CH}_{2} \mathrm{Cl}_{2}(10 \mathrm{ml})$, and then 3,4-dichlorophenylacetic acid (178mg, $0.86 \mathrm{mmol}$ ). To this solution is added 1-[3-(dimethylamino)propyl]-3-ethylcarbodiimide hydrochloride $(217 \mathrm{mg}, 1.13 \mathrm{mmol})$ and dimethylaminopyridine $(10 \mathrm{mg})$. The solution is stirred at room temperature for 16 hours. Water $(20 \mathrm{ml})$ is added and the mixture washed with $\mathrm{CH}_{2} \mathrm{Cl}_{2}(3 \times 30 \mathrm{ml})$. The organic layers are dried $\left(\mathrm{Na}_{2} \mathrm{SO}_{4}\right)$, filtered, and concentrated under reduced pressure. Flash chromatography (50\% EtOAc/ hexanes with $5 \% \mathrm{Et}_{3} \mathrm{~N}$ ) yields the product as an oil $(320 \mathrm{mg}, 90 \%)$. Spectral data was identical to the literature data. ${ }^{6} \mathrm{Rf}=0.1$ on silica gel $(50 \%$ ethyl acetate/hexanes $\left.) ; \alpha\right]^{25}{ }_{\mathrm{D}}=+34.6\left(\mathrm{c}=1.1, \mathrm{CHCl}_{3}\right)$. HRMS calcd for $\mathrm{C}_{15} \mathrm{H}_{22} \mathrm{~N}_{2}\left(\mathrm{MH}^{+}\right)$: 417.1500. Found: 417.1516.

${ }^{6}$ Freeman, J.P.; Michalson, E.T.; D’Andrea, S.V.; Baczynskyj, L.; Von Voigtlander, P.F.; Lahti, R.A.; Smith, M.W.; Lawson, C.F.; Scahill, T.A.; Mizsak, S.A.; Szmuszkovicz, J. J. Med. Chem 1991, 34, 1891. 


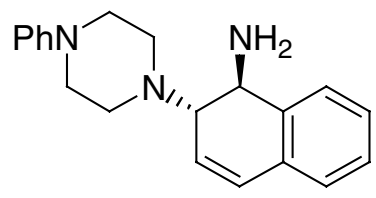

(1S, 2S)-2-(4-Phenyl-piperazin-1-yl)-1,2-dihydro-napthalen-1-ylamine : In a 25ml round bottom flask was added $10(300 \mathrm{mg}, 0.91 \mathrm{mmol}), \mathrm{CH}_{2} \mathrm{Cl}_{2}$, and dropwise additon of trifluoroacetic acid $(1 \mathrm{ml})$. The solution is stirred at room temperature for 45 minutes. $10 \mathrm{ml}$ of $\mathrm{KOH}(10 \%$ aqueous solution) was added and the mixture was washed with $\mathrm{CH}_{2} \mathrm{Cl}_{2}(3 \times 20 \mathrm{ml})$. The organic layers are combined, dried over $\mathrm{Na}_{2} \mathrm{SO}_{4}$, filtered, and concentrated under reduced pressure to yield the product as a white solid $(274 \mathrm{mg}, 99 \%)$. $\mathrm{R}_{\mathrm{f}}=0.1$ on silica gel $\left(100 \%\right.$ ethyl acetate); $\mathrm{mp} 120-123^{\circ} \mathrm{C}\left(\mathrm{Et}_{2} \mathrm{O}\right) ;[\alpha]^{25}{ }_{\mathrm{D}}=+404(\mathrm{c}=1.16$, $\left.\mathrm{CHCl}_{3}\right)$; IR $\left(\mathrm{KBr}, \mathrm{cm}^{-1}\right)$ 3620(m), 3022(s), 2400(s), 1599(m), 1522(s), 1423(s), 1333(w), 1233(s), 1046(s), 928(m), 720(s); ${ }^{1} \mathrm{H}$ NMR (400MHz, $\left.\mathrm{CDCl}_{3}\right) \delta$ 8.40-7.37(1H, m), 7.24(4H, m), 7.08-7.05(1H, m), 6.88-6.78(3H, m), 6.59(1H, dd, J=13.2Hz, $2.4 \mathrm{~Hz})$, $6.03(1 \mathrm{H}, \mathrm{dd}, \mathrm{J}=13 \mathrm{~Hz}, 4.8 \mathrm{~Hz}), 4.12(1 \mathrm{H}, \mathrm{d}, \mathrm{J}=10 \mathrm{~Hz}), 3.33-3.29(1 \mathrm{H}, \mathrm{m}), 3.19-3.07(4 \mathrm{H}, \mathrm{m})$, 2.78-2.63(4H, m), 1.66(2H, s); ${ }^{13} \mathrm{C} \mathrm{NMR}\left(400 \mathrm{MHz}, \mathrm{CDCl}_{3}\right) \delta 151.11,138.86,131.68$, 129.24, 128.91, 127.85, 127.08, 126.50, 125.73, 124.71, 119.59, 116.00, 66.96, 50.08, 49.86, 48.81. HRMS calcd for $\mathrm{C}_{20} \mathrm{H}_{23} \mathrm{~N}_{3}\left(\mathrm{M}^{+}\right)$: 305.1891. Found: 305.1899 .

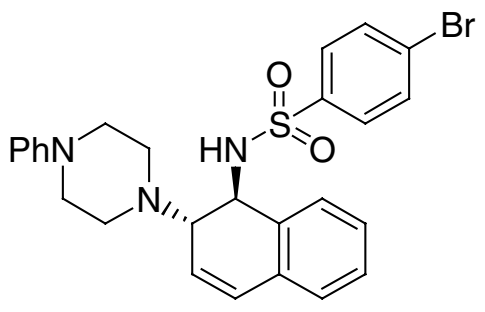

(1S, 2S)-4-Bromo-N-[2-(4-phenyl-piperazin-1-yl)-1,2-dihydro-naphthalen-1-yl]benzenesulfonamide : In a $25 \mathrm{ml}$ round bottom flask was added 2-(4-Phenyl-piperazin1-yl)-1,2-dihydro-napthalen-1-ylamine (300mg, $0.91 \mathrm{mmol}), \mathrm{CH}_{2} \mathrm{Cl}_{2}, \mathrm{Et}_{3} \mathrm{~N}$, and 4bromobenzenesulfonyl chloride. The solution is stirred at room temperature for 16 hours. $10 \mathrm{ml}$ of $\mathrm{KOH}(10 \%$ aqueous solution) was added and the mixture was washed with $\mathrm{CH}_{2} \mathrm{Cl}_{2}(3 \times 20 \mathrm{ml})$. The organic layers are combined, dried over $\mathrm{Na}_{2} \mathrm{SO}_{4}$, filtered, and concentrated under reduced pressure to yield the product as a white solid $(274 \mathrm{mg}, 99 \%)$. $\mathrm{R}_{\mathrm{f}}=0.4$ on silica gel $\left(20 \%\right.$ ethyl acetate/hexanes); mp $188-190^{\circ} \mathrm{C}\left(\right.$ EtOAc); $[\alpha]^{25}{ }_{\mathrm{D}}=$ +224.2( $\left.\mathrm{c}=0.85, \mathrm{CHCl}_{3}\right) ; \mathrm{IR}\left(\mathrm{KBr}, \mathrm{cm}^{-1}\right)$ 3387(w), 3014(s), 2400(m), 2046(w), 1968(w), 1912(w), 1600(w), 1522(s), 1422(s), 1331(m), 1202(s), 1045(s), 929(s), 795(s); ${ }^{1} \mathrm{H}$ NMR $\left(400 \mathrm{MHz}, \mathrm{CDCl}_{3}\right) \delta$ 7.74-7.72(4H, m), 7.26-7.21(4H, m), 7.11-7.07(2H, m), 6.85$6.82(3 \mathrm{H}, \mathrm{m}), 6.78(1 \mathrm{H}, \mathrm{d}, \mathrm{J}=8 \mathrm{~Hz}), 6.69(1 \mathrm{H}, \mathrm{d}, \mathrm{J}=10 \mathrm{~Hz}), 5.95(1 \mathrm{H}, \mathrm{dd}, \mathrm{J}=9.8 \mathrm{~Hz}, 5.2 \mathrm{~Hz})$, 4.68-4.66 $(1 \mathrm{H}, \mathrm{m}), 4.62(1 \mathrm{H}, \mathrm{s}), 3.55-3.53(1 \mathrm{H}, \mathrm{m}), 3.04-2.97(4 \mathrm{H}, \mathrm{m}), 2.68-2.63(2 \mathrm{H}, \mathrm{m})$, 2.47-2.42(2H, m); $\left.{ }^{13} \mathrm{C} \mathrm{NMR} \mathrm{(400MHz,} \mathrm{CDCl}_{3}\right) \delta 151.38,140.15,133.41,132.58$, 132.26, 130.07, 129.23, 128.95, 128.63, 127.98, 122.82, 127.82, 127.25, 124.47, 120.06, 116.38, 63.78, 51.44, 49.76, 48.65. HRMS calcd for $\mathrm{C}_{25} \mathrm{H}_{26} \mathrm{~N}_{3} \mathrm{O}_{2} \mathrm{SBr}\left(\mathrm{M}^{+}\right)$: 523.0929. Found: 523.0908 . 